\title{
„Heute komm ich mal mit meinem Bein ...“
}

\section{„I Came Today Because of my Leg...“}

\author{
Autor \\ W. Koenen \\ Institut \\ Klinik für Dermatologie, Venerologie und Allergologie, Universitätsklinikum Mannheim
}

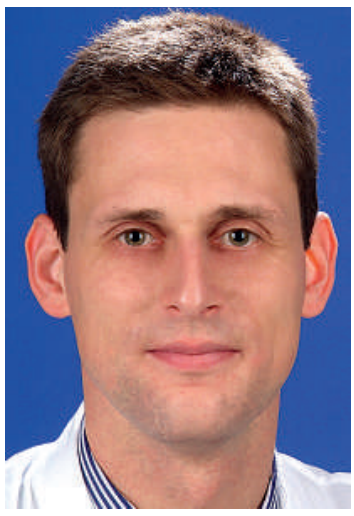

Dr. Wolfgang Koenen
Dieser launige Titel des Kollegen und Kabarettisten Dr. Ludger Stratmann zog Mitte der neunziger Jahre viele Tausend Besucher in seine Vorstellungen im Essener Europa-Haus. Hypochonder Jupp berichtete von seinen diversen Zipperlein und strapazierte die Lachmuskeln des Publikums. Patienten mit Beinleiden gehören für viele von uns zum klinischen Alltag dazu, nur werden weniger unsere Lachmuskeln strapaziert. Ulcera cruris sind eine weit verbreitete Erkrankung unterschiedlicher Genese, deren Behandlung Jahr für Jahr mit mehreren Milliarden Euro zu Buche schlägt.

Die häufigste Erkrankungsursache sind Krankheiten des venösen Formenkreises, deutlich seltener sind es arterielle Ursachen oder eine gemischt arteriell-venöse Genese. Differenzialdiagnostisch dürfen seltene Krankheitsbilder wie Pyoderma gangränosum, Vaskulitiden, ulzerierte Tumoren oder auch Artefakte nicht unberücksichtigt bleiben.

Das vorliegende Heft greift mehrere interessante Aspekte dieses Themenkomplexes auf: Wie entstehen Ulcera cruris? Wie häufig kommen sie vor, welche Kosten verursachen sie? Welche Differenzialdiagnosen kommen in Betracht? Aktuelle Behandlungsstandards für arterielle und gemischte Ulzera aus gefäßchirurgischer Sicht finden Eingang, ebenso die überarbeiteten Empfehlungen der Deutschen Gesellschaft für Phlebologie mit einer Leitlinie zum Ulcus cruris venosum. Die Wundtherapie mit niederfrequentem Ultraschall ist sicherlich noch wenig verbreitet, wissenschaftliche Grundlagen erarbeitet ein weiterer Artikel.

Die Behandlung chronischer Wunden, speziell von Ulcera cruris erfordert Umsicht, Erfahrung und Wissen. Das breite Angebot verschiedenster Wundtherapeutika und neuer Behandlungsmethoden macht es schwer, klar strukturierte Behandlungspfade zu erarbeiten und einzuhalten. Die Kollegen, die für die folgenden Artikel gewonnen werden konnten, berichten vom aktuellen medizinischen Stand und haben die wichtigsten Informationen strukturiert aufbereitet. Ihnen gilt mein spezieller Dank für ihre Bereitschaft, bei der Erstellung dieses Heftes mitzuwirken.

\footnotetext{
Bibliografie

DOI $10.1055 / \mathrm{s}-0029-1214628$

Akt Dermatol 2009; 35:

211 (c) Georg Thieme

Verlag KG Stuttgart · New York

ISSN 0340-2541

Korrespondenzadresse

Dr. med. Wolfgang Koenen

Klinik für Dermatologie

Venerologie und Allergologie

Universitätsklinikum Mannheim

Theodor-Kutzer-Ufer 1 - 3

68135 Mannheim

wolfgang.koenen@

haut.ma.uni-heidelberg.de
} 